\title{
Exercise reduces systemic immune inflammation index (SII) in childhood cancer patients
}

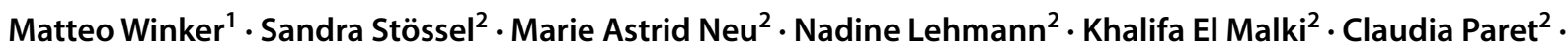 \\ Niklas Joisten ${ }^{3} \cdot$ Wilhelm Bloch ${ }^{1} \cdot$ Philipp Zimmer $^{3}$ (1) Jörg Faber ${ }^{2}$
}

Received: 29 June 2021 / Accepted: 22 November 2021 / Published online: 3 December 2021

(C) The Author(s) 2021

\begin{abstract}
While exercise and physical activity have been suggested to reduce mortality and symptoms in cancer, knowledge on these associations in patients with childhood cancer (CCPs) is sparse. Anti-inflammatory properties of exercise might mediate these beneficial effects. We investigated the influence of exercise on the inflammation markers neutrophil-to-lymphocyte ratio, platelet-to-lymphocyte ratio, and systemic-immune-inflammation index (SII) and associations to patient-reported-outcomes in CCPs in a randomized-controlled trial. Results show associations between inflammation markers and patient-reported outcomes. Compared to the control group, SII was significantly reduced following exercise $(p=0.036)$. Anti-inflammatory effects of exercise are also present in CCPs and may underlie exercise-induced benefits on symptoms. Clinical Trial Registration Number: NCT02612025
\end{abstract}

Keywords Tumor entities $\cdot$ Cellular inflammation $\cdot$ Pediatric oncology $\cdot$ Resistance training $\cdot$ Endurance training

\section{Introduction}

In adults, exercise and physical activity reduce cancer mortality [1] as well as treatment-related side effects, such as depression or fatigue $[2,3]$. Some of these beneficial properties have been suggested to be mediated by the anti-inflammatory environment which is promoted through regular exercise [1,4]. Recently, research approaches have tried to transfer these promising findings to the childhood cancer setting $[5,6]$.

Matteo Winker and Sandra Stössel shared first authorship.

Philipp Zimmer and Jörg Faber shared last authorship.

Philipp Zimmer

philipp.zimmer@tu-dortmund.de

1 Department for Molecular and Cellular Sports Medicine, Institute of Cardiovascular Research and Sports Medicine, German Sport University, Cologne, Germany

2 Center for Pediatric and Adolescent Medicine, Childhood Cancer Center, University Medical Center Mainz, Mainz, Germany

3 Department of "Performance and Health (Sports Medicine)", Institute of Sport and Sport Science, Technical University Dortmund, Otto-Hahn-Straße 3, 44227 Dortmund, Germany
Inflammation contributes to different chronic diseases and is associated with several disease-specific symptoms in patients with childhood cancer (CCPs) [7, 8]. In the past years, the cellular immune inflammation markers neutrophilto-lymphocyte ratio (NLR), platelet-to-lymphocyte ratio (PLR), and systemic immune-inflammation index (SII) have been proofed to be reliable determinants of inflammatory responses. Indeed, several studies confirmed the prognostic value of all three markers with regard to progression [9], symptoms [3], and survival [1] in different cancer diseases. These promising and easily traceable markers were shown to be reduced by exercise in patients with multiple sclerosis [10].

Since knowledge on the benefits of exercise training in CCPs is sparse, an investigation of potentially underlying mechanisms would be helpful to improve populationspecific exercise recommendations. Here, we exploratory investigated potential associations of NLR, PLR, and SII, with disease- and treatment-related symptoms and whether an exercise intervention decreases these markers in CCPs. We hypothesize that NLR, PLR, and SII are associated with different symptoms such as fatigue and quality of life $(\mathrm{QoL})$ and that an exercise intervention decreases these markers in CCPs. 


\section{Methods}

In this secondary analysis of a randomized controlled trial, NLR, PLR, and SII from 25 CCPs undergoing cancer treatment aged 4-17 years (Table 1) were determined from venous blood samples. Participants were allocated to an exercise group (EG) or a control group (CG) using stratified randomization with age, sex, tumor entity, and physical performance status as stratification factors. The study was approved by the regional ethical committee. A detailed description of the primary study has been published elsewhere [11]. In brief, the EG participated in a 6-8-week supervised exercise intervention including three exercise sessions per week (45-60min) of patient-adapted, moderate intensity endurance and strength exercises. The timeframe of intervention varied since all exercise tests were conducted prior to a new cancer therapy cycle on condition of hematologic recovery to ensure comparability. Training load was adjusted based on the results of the 6-min walking test as well as a questionnaire asking for physical activity level and intensity in daily life, prior to disease onset [12]. During the intervention, intensity was considered as moderate if two of the following criteria were fulfilled: A score of 12 to 13 on the Borg Scale, $60-75 \%$ of estimated maximum heart rate, a maximum of three repetitions with correct technical execution, and a rating by the supervisor based on physiological parameters [13]. The CG received anti-cancer treatment recommended by their medical supervisor. To evaluate the level of fatigue, the PedsQL3.0 questionnaire was used [14]. The QoL was determined with the KINDL questionnaire [15]. Parent-report versions of both German language questionnaires were used. Venous blood samples were collected during a resting condition before the first exercise session (baseline) and after the last exercise session (post).

Subjects were included if at least one blood sample was collected. NLR, SII, and PLR were calculated from hemograms using an automated hematology analyzer (Sysmex xs-800i von Sysmex Deutschland GmbH). Data was winsorized to $z$-scores of three [16] and tested for normality using Kolmogorov-Smirnoff test. Since normality could not be assumed for most of the variables, we $\log 10$ transformed NLR, SII, and PLR at both measurement time points. Spearman's rank correlation coefficient was calculated to investigate potential associations between the cellular inflammation markers and quality of life and fatigue in CCPs. To evaluate the effects of the exercise intervention on NLR, SII, and PLR, delta values of EG versus CG were compared using a one-way analysis of variance (ANOVA). The level of significance was set at $p \leq 0.05$. Statistical analysis was performed using IBM SPSS Statistics software Version 27 (IBM, Armonk, NY, USA).

\section{Results}

Fatigue was negatively correlated with NLR $(r=-0.702$; $p=0.001)$, PLR $(r=-0.486 ; p=0.030)$, and SII $(r=-0.742$; $p<0.001)$ at baseline. QoL negatively correlated with NLR $(r=-0.442 ; p=0.035)$ and SII $(r=-0.452 ; p=0.030)$ at baseline (Fig. 1A). No significant correlation was observed between QoL and PLR ( $r=-0.074 ; p=0.737)$ at baseline. Graphical illustrations of associations between NLR, PLR, SII, and fatigue as well as QoL are depicted in Fig. 1A. Regarding exercise intervention effects, delta value comparisons showed a significant between group effect for SII ( $\mathrm{df}=1$, $F=4.963, p=0.036$ ). While the exercise intervention reduced
Table 1 Participants' characteristics separated by exercise intervention

\begin{tabular}{lllll}
\hline & EG $(n=11)$ & CG $(n=14)$ & Overall $(n=25)$ & $p$-value \\
\hline Sex (f/m) & $5 / 6$ & $7 / 7$ & $12 / 13$ & - \\
Age at diagnosis (years) & $11.19 \pm 5.60$ & $11.28 \pm 4.41$ & $11.24 \pm 4.86$ & 0.964 \\
Bodyweight (kg) & $46.00 \pm 21.52$ & $39.86 \pm 17.97$ & $42.56 \pm 19.43$ & 0.445 \\
Tumor entity (AL or TCL/CNS/others) & $6 / 1 / 4$ & $5 / 2 / 7$ & $11 / 3 / 11$ & - \\
6-min walk test (m) & $450.17 \pm 164.86$ & $543.66 \pm 76.16$ & $502.52 \pm 129.26$ & 0.072 \\
Fatigue at baseline (score) & $61.53 \pm 20.37$ & $71.52 \pm 7.43$ & $67.03 \pm 15.16$ & 0.193 \\
QoL at baseline (total score) & $71.18 \pm 8.76$ & $71.91 \pm 14.57$ & $71.63 \pm 12.39$ & 0.893 \\
Duration of treatment (weeks) & $8.04 \pm 1.45$ & $8.42 \pm 2.90$ & $8.25 \pm 2.32$ & 0.700 \\
Exercise sessions (numbers) & $19.93 \pm 8.85$ & - & - & - \\
NLR at baseline & $1.54 \pm 0.92$ & $1.26 \pm 1.09$ & $1.39 \pm 1.00$ & 0.504 \\
PLR at baseline & $0.30 \pm 0.20$ & $0.33 \pm 0.33$ & $0.31 \pm 0.27$ & 0.825 \\
SII at baseline & $564.52 \pm 412.43$ & $737.22 \pm 975.53$ & $665.26 \pm 782.31$ & 0.605 \\
\hline
\end{tabular}

$E G$, exercise group; $C G$, control group; $f$, female; $m$, male; $A L$, acute leukemia; $T C L$, T-cell lymphoma; $C N S$, central nervous system tumor; Fatigue [total score] evaluated with PedsQL 3.0 questionnaire by parents; QoL [total score] evaluated with KINDL questionnaire by parents; $N L R$, neutrophil-to-lymphocyte ratio; $P L R$, platelet-to-lymphocyte ratio; SII, systemic immune-inflammation index 
(a) Relationship between cellular inflammation markers and patient reported outcomes at baseline (T0)

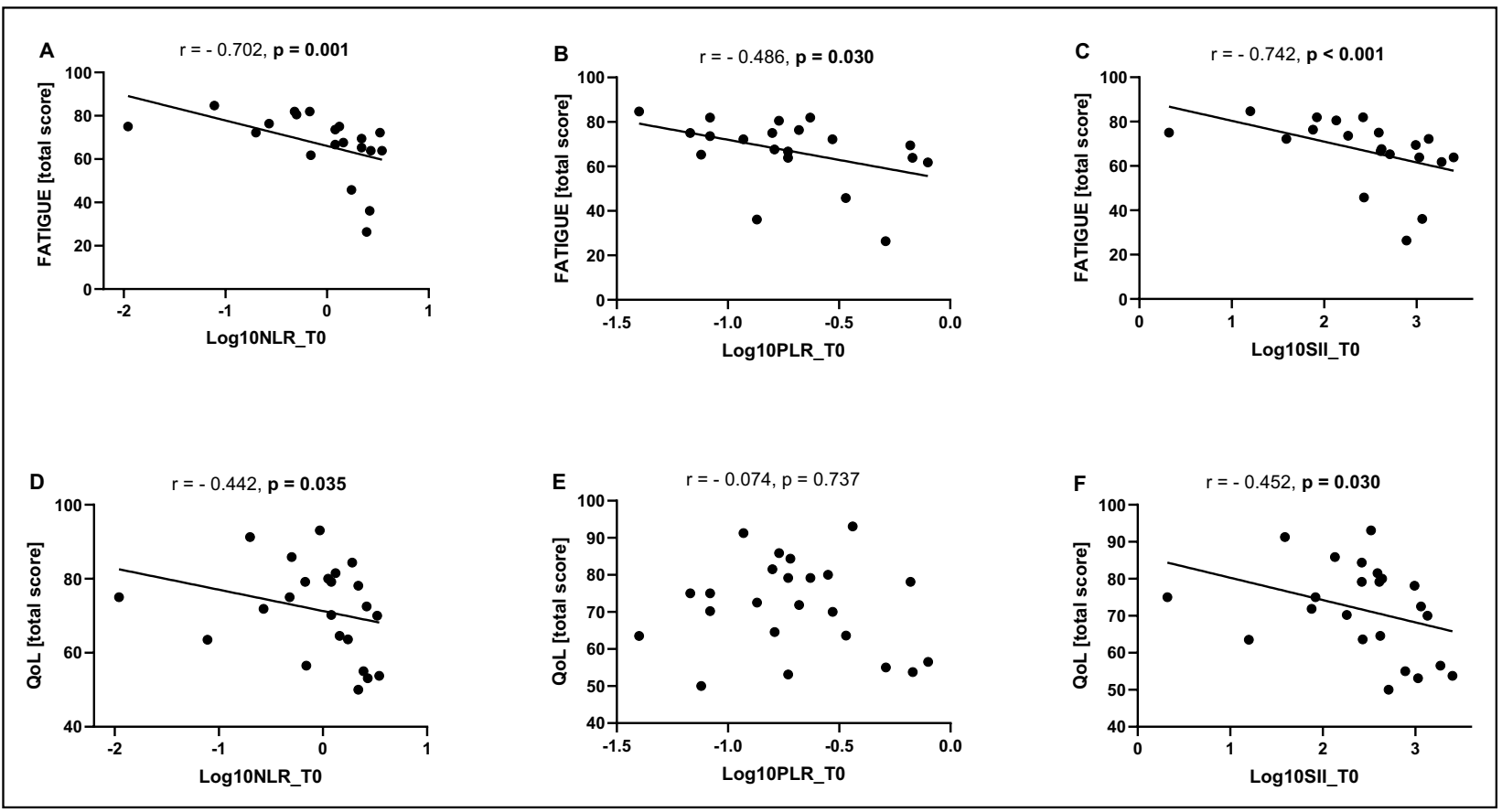

(b) Exercise intervention effects indicated as delta values seperated by groups
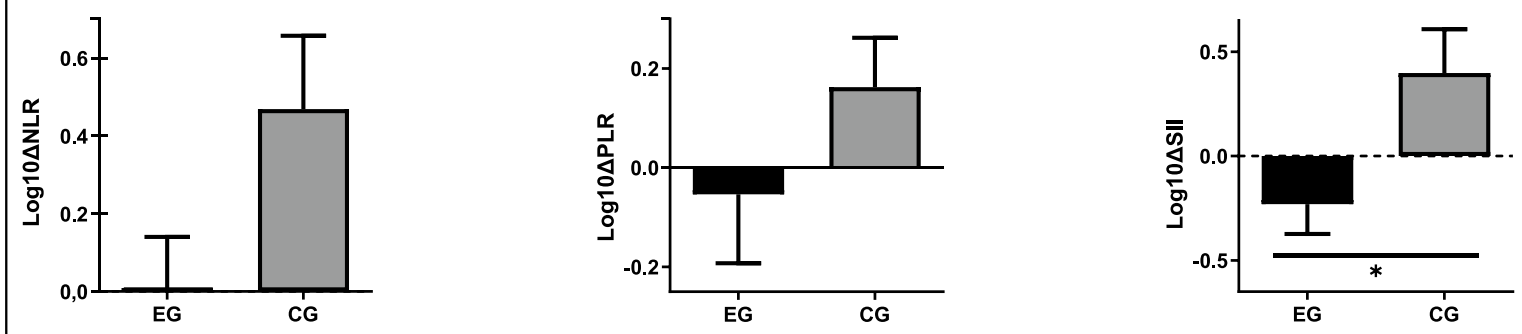

Fig. 1 Correlation analysis (a) of fatigue with $\log 10$-transformed data of (A) NLR, (B) PLR, (C) SII at baseline, as well as QoL with Log10-transformed data of (D) NLR, (E) PLR, and (F) SII at baseline. Log10-transformed data (b) of training effects from pre- (baseline) to post-intervention illustrated as delta values. NLR, neutrophilto-lymphocyte ratio; PLR, platelet-to-lymphocyte ratio; SII, systemic

SII, an increase was observed for the CG. No significant effects between the groups were observed for NLR $(\mathrm{df}=1$, $F=3.605, p=0.070)$ and PLR $(\mathrm{df}=1, F=0.484, p=0.493)$.

\section{Discussion}

The results of this secondary analysis of the MUCKI trial confirm findings from studies in adults reporting associations between the cellular inflammation markers NLR, PLR, immune inflammation index; $\log 10 \_$T0, $\log 10$-transformed data at baseline. Fatigue [total score] evaluated with PedsQL 3.0 questionnaire by parents; QoL [total score] evaluated with KINDL questionnaire by parents. Values are presented as mean \pm standard error. *Significant between-group effect

SII, and fatigue as well as QoL [3, 11, 17, 18]. Moreover, our results provide first evidence that a physical exercise intervention in CCPs was able to reduce SII.

From a descriptive point of view, the NLR shows an inverse development during the intervention period between the two groups, with a downregulation in the EG and an increase in the CG. However, statistical significance was only observed between the two groups for SII. The exerciseinduced reduction of SII highlights the anti-inflammatory effects of exercise, which have been proposed to mediate the 
beneficial effects of exercise training on various symptoms in cancer patients $[2,19]$. The present study suggests similar effects of exercise in CCPs.

The small sample size $(n=25)$ and heterogeneity in included tumor entities represent central limitations of the investigation and may led to absent statistical between group differences of the NLR.

In conclusion, results of the present study confirm that the well-described anti-inflammatory effects of exercise are also present in CCPs $[2,5,10]$. Considering the exploratory character of this analysis, the reported findings cannot be generalized, and future powered randomized controlled trials are needed to confirm our promising findings and highlight the clinical relevance.

Funding Open Access funding enabled and organized by Projekt DEAL.

\section{Declarations}

Conflict of interest The authors declare no competing interests.

Open Access This article is licensed under a Creative Commons Attribution 4.0 International License, which permits use, sharing, adaptation, distribution and reproduction in any medium or format, as long as you give appropriate credit to the original author(s) and the source, provide a link to the Creative Commons licence, and indicate if changes were made. The images or other third party material in this article are included in the article's Creative Commons licence, unless indicated otherwise in a credit line to the material. If material is not included in the article's Creative Commons licence and your intended use is not permitted by statutory regulation or exceeds the permitted use, you will need to obtain permission directly from the copyright holder. To view a copy of this licence, visit http://creativecommons.org/licenses/by/4.0/.

\section{References}

1. Ahn HK, Hwang IC, Lee JS et al (2016) Neutrophil-lymphocyte ratio predicts survival in terminal cancer patients. J Palliat Med 19:437-441. https://doi.org/10.1089/jpm.2015.0277

2. Pagola I, Morales JS, Alejo LB et al (2020) Concurrent exercise interventions in breast cancer survivors with cancer-related fatigue. Int J Sports Med 41:790-797. https://doi.org/10. 1055/a-1147-1513

3. McFarland D, McFarland D (2020) Neutrophil to lymphocyte ratio in lung cancer: implications for depressive symptoms and survival. Clin Oncol Res 1-7. https://doi.org/10.31487/j.cor.2020. 06.12

4. Wang D, Guo D, Shi F et al (2019) The predictive effect of the systemic immune-inflammation index for patients with small-cell lung cancer. Future Oncol 15:3367-3379. https://doi.org/10.2217/ fon-2019-0288

5. Yeh CH, Man Wai JP, Lin US, Chiang YC (2011) A pilot study to examine the feasibility and effects of a home-based aerobic program on reducing fatigue in children with acute lymphoblastic leukemia. Cancer Nurs 34:3-12. https://doi.org/10.1097/NCC. 0b013e3181e4553c
6. Tanir MK, Kuguoglu S (2013) Impact of exercise on lower activity levels in children with acute lymphoblastic leukemia: a randomized controlled trial from Turkey. Rehabil Nurs 38:48-59. https://doi.org/10.1002/rnj.58

7. Libby P (2007) Inflammatory mechanisms: the molecular basis of inflammation and disease. Nutr Rev 65. https://doi.org/10.1111/j. 1753-4887.2007.tb00352.x

8. Cheung YT, Brinkman TM, Mulrooney DA et al (2017) Impact of sleep, fatigue, and systemic inflammation on neurocognitive and behavioral outcomes in long-term survivors of childhood acute lymphoblastic leukemia. Cancer 123:3410-3419. https://doi.org/ 10.1002/cncr.30742

9. Stojkovic Lalosevic M, Pavlovic Markovic A, Stankovic S et al (2019) Combined diagnostic efficacy of neutrophil-to-lymphocyte ratio (NLR), platelet-to-lymphocyte ratio (PLR), and mean platelet volume (MPV) as biomarkers of systemic inflammation in the diagnosis of colorectal cancer. Dis Markers 2019:6036979. https:// doi.org/10.1155/2019/6036979

10. Joisten N, Proschinger S, Rademacher A et al (2020) High-intensity interval training reduces neutrophil-to-lymphocyte ratio in persons with multiple sclerosis during inpatient rehabilitation. Mult Scler J 1-4. https://doi.org/10.1177/1352458520951382

11. Stössel S, Neu MA, Wingerter A et al (2020) Benefits of exercise training for children and adolescents undergoing cancer treatment: results from the randomized controlled MUCKI trial. Front Pediatr 8:1-10. https://doi.org/10.3389/fped.2020.00243

12. Opper E, Wagner M, Bös K (2007) Motorik-Modul (MoMo) im Rahmen des Kinder-und Jugendgesundheitssurveys (KiGGS). Bundesgesundheitsbl Gesundheitsforsch Gesundheitsschutz 50:879-888. https://doi.org/10.1007/s00103-007-0251-5

13. Borg G (2004) Anstrengungsempfinden und körperliche Aktivität. Dtsch Arztebl 101:1016-1021

14. Jung M, Höhne A, Varni J et al (2009) Die Messung von Fatigue in der pädiatrischen Onkologie: Die "PedsQL 3.0 Multidimensional Fatigue Scale" in einer ersten deutschen Version. Kinderund Jugendmedizin 09:218-224. https://doi.org/10.1055/s-00381629061

15. Ravens-Sieberer U, Bullinger M (1998) Assessing health-related quality of life in chronically ill children with the German KINDL: first psychometric and content analytical results. Qual Life Res 7:399-407. https://doi.org/10.1023/A:1008853819715

16. Kwak SK, Kim JH (2017) Statistical data preparation: management of missing values and outliers. Korean J Anesthesiol 70:407-411. https://doi.org/10.4097/kjae.2017.70.4.407

17. Sunbul EA, Sunbul M, Yanartas O et al (2016) Increased neutrophil/lymphocyte ratio in patients with depression is correlated with the severity of depression and cardiovascular risk factors. Psychiatry Investig 13:121-126. https://doi.org/10.4306/pi.2016. 13.1.121

18. Wang L, Wang C, Wang J et al (2017) A novel systemic immuneinflammation index predicts survival and quality of life of patients after curative resection for esophageal squamous cell carcinoma. J Cancer Res Clin Oncol 143:2077-2086. https://doi.org/10.1007/ s00432-017-2451-1

19. Hojan K, Kowska-Borowczyk EK, Leporowska E et al (2016) Physical exercise for functional capacity, blood immune function, fatigue, and quality of life in high-risk prostate cancer patients during radiotherapy: a prospective, randomized clinical study. Eur J Phys Rehabil Med 52:489-501

Publisher's note Springer Nature remains neutral with regard to jurisdictional claims in published maps and institutional affiliations. 